\title{
Fully three-dimensional analysis of a photonic crystal assisted silicon on insulator waveguide bend
}

\author{
N. Eti* \\ Department of Mathematics, Izmir Institute of Technology, \\ Gulbahce Kampus 35330, Urla, Izmir, Turkey \\ neslihaneti@gmail.com \\ Z. Çetin* and H. S. Sözüer ${ }^{\dagger}$ \\ Department of Physics, Izmir Institute of Technology, \\ Gulbahce Kampus 35330, Urla, Izmir, Turkey \\ *zebihcetin@iyte.edu.tr \\ †samisozuer@iyte.edu.tr
}

Received 7 June 2018

Revised 7 October 2018

Accepted 16 October 2018

Published 19 December 2018

\begin{abstract}
A detailed numerical study of low-loss silicon on insulator (SOI) waveguide bend is presented using the fully three-dimensional (3D) finite-difference time-domain (FDTD) method. The geometrical parameters are optimized to minimize the bending loss over a range of frequencies. Transmission results for the conventional single bend and photonic crystal assisted SOI waveguide bend are compared. Calculations are performed for the transmission values of TE-like modes where the electric field is strongly transverse to the direction of propagation. The best obtained transmission is over $95 \%$ for TE-like modes.
\end{abstract}

Keywords: Silicon-on-insulator; integrated optics; optical waveguides.

PACS numbers: 42.70.Qs, 85.40.-e, 78.67.Pt

\section{Introduction}

Silicon on insulator (SOI) waveguides and waveguide bends have been studied widely over the years, and are used to control light propagation in integrated optical circuit designs. ${ }^{1-7}$ Two-dimensional (2D) modeling of these waveguides allows great ease of computational burden, and therefore the 2D approach has been adopted widely, although at the expense of some accuracy. ${ }^{8-10}$ Despite the high computational cost, in this work we use fully three-dimensional (3D) calculations to determine the optimal structure for a photonic crystal assisted $90^{\circ}$ waveguide bend.

\footnotetext{
${ }^{*}$ Corresponding author.
} 
3D waveguides can be realized using a variety of geometries. The most effective way appears to be by "drilling" channels in fully $3 \mathrm{D}$ photonic crystals such as the fcc inverse opal ${ }^{11}$ or woodpile ${ }^{12-16}$ or other types of $2 \mathrm{D}-3 \mathrm{D}$ photonic crystals. ${ }^{17,20-24}$ In recent years, there has been extensive research on photonic crystal $(\mathrm{PhC})$ waveguide slabs. Zhang and Feng ${ }^{24}$ explored the optimization of waveguide bends in $2 \mathrm{D}+3 \mathrm{D}$ hetero-structures composed of $2 \mathrm{D}$ and woodpile structure. They used both smoothing optimization and periodicity changing to improve the transmission. The best transmission result was around $95 \%$. Chutinan et al., ${ }^{20}$ employed an effective index approximation to simplify guide bend with additional air holes from $3 \mathrm{D}$ to $2 \mathrm{D}$ and by making the waveguide single mode at the bend. They found the transmission around $90 \%$. In another work, Cai et al., $\frac{17}{17}$ used FDTD modeling and a simple perfect mirror model analysis and found the bend transmission around $97.3 \%$. Also optimization of waveguide bends in $2 \mathrm{D}$ has been demonstrated broadly and versatile solutions have been suggested. It is shown that by using a $2 \mathrm{D}$ square lattice of annular dielectric rods, a polarization-independent single-mode waveguide bend is established. The optimizations have obtained changing the dimensions and positions of the corner elements. $\frac{18}{}$ Another work, $\frac{19}{2}$ used an inverse design technique to make the optimization of very sharp adiabatic bends and fabricated the device on a SOI platform.

The practical difficulty in manufacturing 3D photonic crystals with these complicated geometries led researchers to consider "2D-like" waveguides such as ridge silicon on silica insulator substrate, where containment is provided by the refractive index. However, the high loss at bends meant there was a need to use photonic crystals to avoid bending losses. ${ }^{25,26}$

In this study using a minimalist approach we adopt ridge waveguides for straight sections, and photonic crystal assistance at corners where bending loss is an issue. The designed $\mathrm{PhC}$ waveguide slabs have simple $3 \mathrm{D}$ geometry, consisting of a thin guiding layer sandwiched between two bounding regions. ${ }^{27,28}$ The guiding layer generally is formed from photonic crystal with a finite thickness in the vertical $z$ direction. Light confinement in the $x y$-plane is achieved via total internal reflection in the straight sections, while the photonic bandgap of the photonic crystal is employed at the bend. Light confinement in the vertical direction is due to the refractive index contrast between the core and the surrounding medium. The SOI waveguide bend we present in this work is capable of bending light through $90^{\circ}$ with a transmittance higher than $95 \%$. The calculations are performed by using the 3D FDTD method.

The organization of the rest of the paper is as follows. In Sec. 2, we present the $2 \mathrm{D}$ and $3 \mathrm{D}$ designs of the structure, and analyze the band diagrams. In Sec. 3, we discuss the 3D FDTD real-time simulation results. In Sec. 4, we investigate enhancement of the transmission as a function of the number of cylinders on either side of the core in the corner element. A brief comparison of $2 \mathrm{D}$ versus $3 \mathrm{D}$ calculations is included in Sec. 5 . Finally, in Sec. 6, we present our conclusions. 


\section{The Design of the SOI Waveguide Bend}

The SOI waveguide bend is a combination of a ridge waveguide and a corner element. The reasoning for this combination is explained as follows: Unlike in the $\mathrm{PhC}$ waveguide, the ridge waveguide is not in a "PhC cladding," which results in a much simpler geometry, largely free from possible manufacturing imperfections ${ }^{29,30}$ that can substantially increase the attenuation in a $\mathrm{PhC}$ waveguide ${ }^{31,32}$ with a complex geometry. Photonic crystal assistance is exploited only when it is absolutely necessary, i.e., at the corner where the ridge waveguide would exhibit a substantial bending loss. The $\mathrm{PhC}$ at the corner element is designed such that, at the operating frequency, it has a bandgap which largely prohibits leakage, resulting in a very small bending loss. The 3D geometry of proposed SOI waveguide bend is shown in Fig. 1.

The FDTD method ${ }^{33}$ is extensively used for studying the propagation of electromagnetic waves through complex structures. We use a freely available software package ${ }^{34,35}$ to perform simulations and calculate the transmission and losses at the various stages of the design. The software package computes fully-vectorial eigenmodes of Maxwell's equations with periodic boundary conditions by preconditioned conjugate-gradient minimization of the block Rayleigh quotient in a planewave basis.

One important distinction between the 2D and the 3D approach is that, while in $2 \mathrm{D}$ calculations the modes neatly split into Transverse Electric (TE) and Transverse Magnetic (TM) types, this is no longer the case in 3D. ${ }^{a}$ Modes can be "TE-like" with a small longitudinal electric field component, or "TM-like" with a small longitudinal magnetic field component. But there are also modes which are of neither type: i.e., modes where both the longitudinal electric field and the longitudinal magnetic field

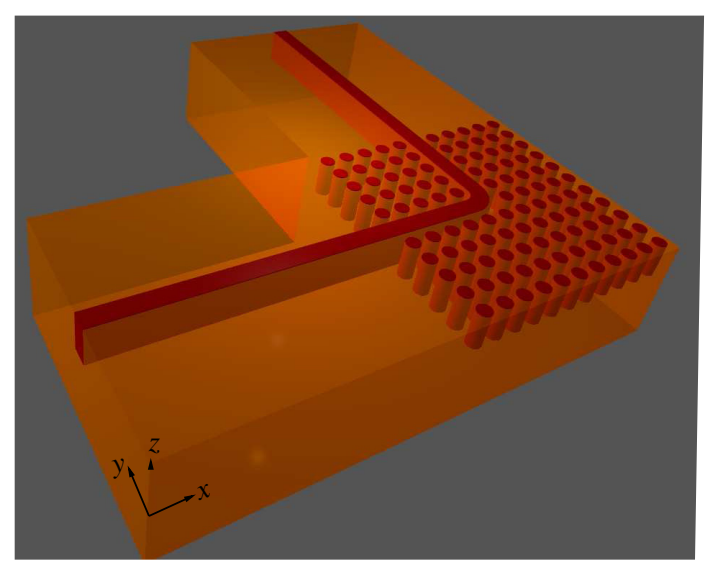

Fig. 1. (Color online) The 3D view of proposed SOI waveguide bend.

\footnotetext{
"We take "transverse" to mean transverse to the direction of propagation of the wave, and not transverse to the axis of cylinders in the photonic crystal.
} 
components are large compared to their transverse counterparts. In this study, "TElike" guided modes are determined by analyzing the longitudinal and transverse components of the electric and the magnetic fields of each and every mode for each structure. The proportion of the small longitudinal component is around 0.01-0.02 compared to transverse components.

We first consider SOI waveguide bend design in 2D and then we study the design in $3 \mathrm{D}$ for the next two subsections.

\subsection{SOI waveguide bend design in $2 D$}

The SOI waveguide bend design in $2 \mathrm{D}$ is displayed in Fig. 2. Figure 2(a) is a cross-section of the fully 3D structure as shown in Fig. 1 at vertical axis $z=0$. The ratio in the parameters of the device structure ( $d, R, \mathrm{Rbend})$ is used from the previous work, $\frac{36}{6}$ to minimize the bending loss in $x y$-plane. Figure $2(\mathrm{~b})$ shows the band diagram of the structure. It is displayed for transverse electric (TE) modes, for which there is no electric field in the direction of propagation (in our case $E_{x}=0$ ).

Since our proposed structure is a combination of the ridge waveguide and the corner element, we must ensure that light should be able to propagate in both parts. In order to have minimal reflection at the interface, we adjust the parameters of the straight segment and the corner element so that the lowest branch of the ridge waveguide bands (shown in green) overlaps almost completely with the

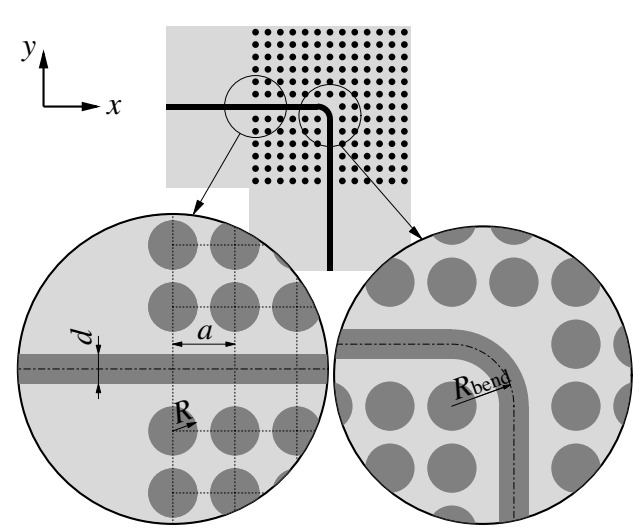

(a)

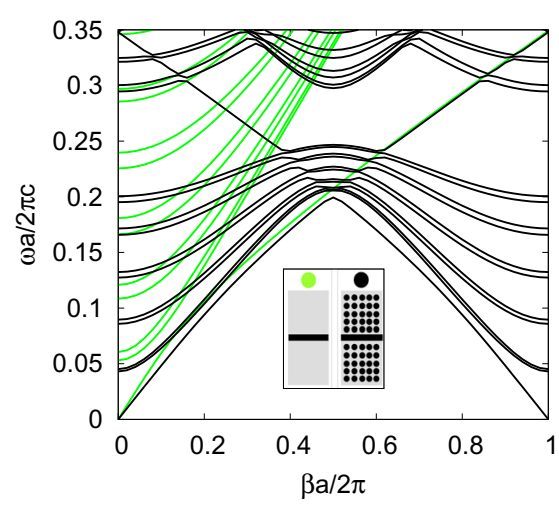

(b)

Fig. 2. (Color online) (a) 2D cross-sectional view of the proposed SOI waveguide bend. Dark regions are silicon with $\epsilon_{a}=13$, and the background medium is silica with $\epsilon_{b}=2.25$. The dimensions are $d=0.3184 a, R=0.2387 a, R_{\text {bend }}=2 d$, where $a$ is the lattice constant. (b) The band diagrams for the SOI waveguide bend in $2 \mathrm{D}$. The band structure of the ridge waveguide, shown in green, is modeled using the supercell method with a supercell size of $A_{y}=11 a$. The band structure of the corner element, shown in black, is modeled using a supercell size of $A_{x} \times A_{y}$, where $A_{x}=a$ and $A_{y}=11 a . \beta$ is the propagation constant in the $x$-direction. The bandgap of the $2 \mathrm{D}$ photonic crystal is in the range $0.24<\omega a / 2 \pi c<0.2903$. The supercell sizes are adjusted to ensure that the guided mode is contained well within the supercell. 
corresponding branch of the corner element (shown in black), as shown in Fig. 2(b). In addition, we must also ensure that the corner element has a bandgap at the operating frequency to keep the guided light from leaking at the corner. The 2D corner element has a bandgap in Fig. 2(b) between the frequencies $0.24<\tilde{\omega}=\frac{\omega a}{2 \pi c}<0.293$. Therefore, the region $0.24<\overline{\tilde{\omega}}=\frac{\omega a}{2 \pi c}<0.293$ and $0.62<\tilde{\beta}=\frac{\beta a}{2 \pi}<0.82$ corresponds to the bandgap of the corner element and the first fundamental guided mode of the ridge waveguide. A similar $\mathrm{PhC}$ waveguide bend in $2 \mathrm{D}$ was previously studied. $\frac{36}{6}$ The obtained transmission result is nearly $100 \%$. In our work, a slice of the $2 \mathrm{D}$ structure with thickness $2 a$ is sandwiched between a silica substrate from below and an air superstrate from above.

\subsection{SOI waveguide bend design in $3 D$}

The SOI waveguide bend in $3 \mathrm{D}$ is displayed in Fig. 1 . It is a structure asymmetrical in the $z$-direction, which rests on a silica substrate, that breaks the mirror symmetry in the vertical direction due to the different dielectric constants of the air superstrate and the silica substrate.

The proposed SOI waveguide bend geometry is displayed in Fig. 1 and the geometrical details are shown in Fig. 3. A thin guiding layer, comprising the ridge waveguide and the corner element, is sandwiched between two bounding regions. The upper and lower bounding regions are air and silica, respectively. The corner element is a square lattice of silicon rods with $\epsilon_{a}=13$, immersed in a silica background with $\epsilon_{b}=2.25$. The line defect for the corner element is constituted by removing one row of dielectric rods and by extending the core of ridge waveguide. The defect is the ridge waveguide with dielectric constant $\epsilon_{d}=13$.

The related geometries and the band diagrams are shown in Fig. 3. The geometrical details of the ridge waveguide and the corner element are given in Figs. 3(a) and $3(\mathrm{~b})$, respectively. The $3 \mathrm{D}$ band diagram of the ridge waveguide is shown in Fig. $\overline{3}(\mathrm{c})$. In the figure the first fundamental mode is guided mode in the line defect. The $3 \mathrm{D}$ band diagram of the ridge waveguide and the corner element are displayed in Fig. 3(d). The big black crosses and green circles show guided modes of the ridge waveguide and the corner element, respectively. The enlarged section of the red marked lines in Fig. 3(d) is shown in terms of group velocities with the black and green lines in Fig. 3(e). The 3D band diagrams of the design are displayed for TE-like modes.

There are two important issues to ensure the guidance of light in SOI waveguide bend design:

(a) The guided modes of the ridge waveguide and the corner element must be in the same frequency range to be coupled

$$
\omega_{\text {ridge }}\left(\beta_{0}\right)=\omega_{\text {corner }}\left(\beta_{0}\right)
$$


(a)

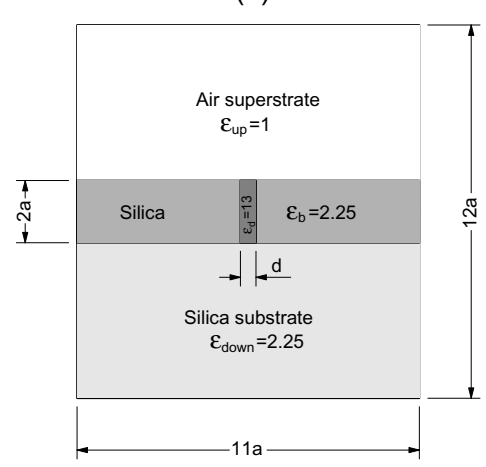

(c)

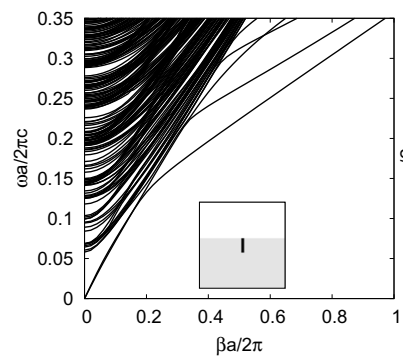

(b)

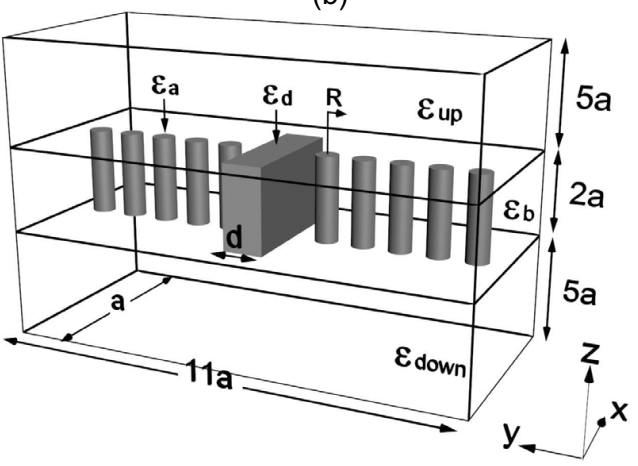

(e)

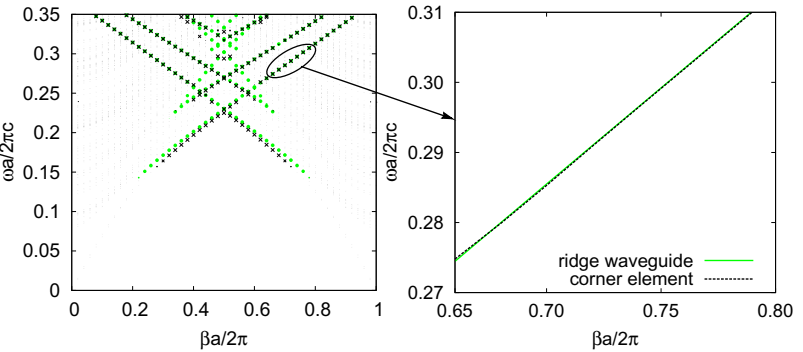

Fig. 3. (Color online) The geometrical details and band diagrams of the SOI waveguide bend. (a) Cross-sectional view of the ridge waveguide, used as the $2 \mathrm{D}$ supercell for calculations of the modes propagating in the $x$-direction. (b) The 3D unit "supercell" of the corner element used for mode calculations in the $+x$-direction. The dielectric constants are as follows; $\epsilon_{a}=\epsilon_{d}=13$, $\epsilon_{b}=\epsilon_{\text {down }}=2.25, \epsilon_{\mathrm{up}}=1$. (c) The $3 \mathrm{D}$ band diagram of the ridge waveguide. (d) The 3D band diagram of the ridge waveguide and the corner element. (e) The enlarged image of (d) for the proposed range of operational frequencies. The near-overlap of the two bands indicates that both the phase and the group velocities of the two match well.

and their group velocities, $v_{g}=\frac{d \omega}{d \beta}$, must match:

$$
\left.\frac{d \omega_{\text {ridge }}}{d \beta}\right|_{\beta_{0}}=\left.\frac{d \omega_{\text {corner }}}{d \beta}\right|_{\beta_{0}} .
$$

(b) The chosen operating frequency must be in the bandgap of the corner element to prevent losses at the $90^{\circ}$ bend.

We performed simulations in the region $0.27<\tilde{\omega}=\frac{\omega a}{2 \pi c}<0.31$ and $0.65<$ $\tilde{\beta}=\frac{\beta a}{2 \pi}<0.80$, where the 2D square lattice corner element has a bandgap, and the dispersion curves of the ridge waveguide and the photonic crystal assisted ridge waveguide nearly overlap, resulting in a good match of both the phase and the group velocities as displayed in Fig. 3(e). Furthermore, the dispersion relation is nearly linear, which would result in little dispersion. These results ensure the guidance of light in SOI waveguide bend with minimal loss at the interface and with little or no dispersion. 


\section{3D FDTD Simulation Results}

We next discuss FDTD simulation results. In the previous section, the calculated band structures provide guidance to determine the optimal design with minimal bending loss for the SOI waveguide bend. However, to actually be sure that the corner design works as expected and determines the actual transmission values, time domain simulations must be performed. Time domain calculations are further necessary for several reasons. First, the band structure of the 3D structure, which is a truncated version of the $2 \mathrm{D}$ structure, would not necessarily have its photonic bandgap in exactly the same range as the bandgap of the 2D structure. Second, the frequency domain calculations are inadequate for predicting the losses due to the curvature of the core at the corner. And third, time domain calculations are required because of possible reflections at the entry and exit interfaces to and from the corner element.

Simulations are performed with the FDTD method, using a freely available software package ${ }^{34}$ which allows great flexibility in using custom sources. Although a realistic source would be a monochromatic dipole source at the entrance of the ridge waveguide, the radiation emitted by a dipole would be a linear combination of many modes of the ridge waveguide, most of which are unguided evanescent modes which penetrate the waveguide but decay exponentially inside with various decay lengths. The length of the ridge waveguide would then need to be very large in order for the various unguided evanescent modes to exit the ridge waveguide before entering the bend with only the propagating mode remaining. This would require a very large simulation region, which is beyond our computational resources. Therefore, we employ a "mode source" which has the spatial profile of the mode of the ridge waveguide at the operating frequency, thus eliminating the need for a long segment where the unguided modes die out. Thus, we first calculate the spatial mode profile of the ridge waveguide at the operating frequency using a plane wave expansion. The time dependence is a Gaussian envelope in time with the operating frequency as the center frequency. This is then used as the current source for the FDTD simulation. It has the form

$$
\mathbf{J}_{\mathbf{m}}(\mathbf{r}, t)=\delta\left(x-x_{s}\right) \mathbf{H}(y, z, \omega) \exp \left[-\frac{\left(t-t_{0}\right)^{2}}{2 \sigma^{2}}\right] \exp (-i \omega t),
$$

where $\mathbf{J}_{\mathbf{m}}(\mathbf{r}, t)$ is a monochromatic source located at $x=x_{\mathrm{s}}$ with frequency $\omega$. It is enveloped in a Gaussian packet with a width of $\Delta \omega=1 / \sigma$, where $\sigma$ is the temporal width. $\mathbf{H}(y, z, \omega)$ is the TE-like guided mode of the ridge waveguide at the center frequency $\omega$. We use $H_{x}(y, z, \omega), H_{y}(y, z, \omega), H_{z}(y, z, \omega)$ to generate a current source in the $y z$-plane in order to excite TE-like modes. For flux calculations, the Gaussian source is kept on until well after the fields decay to 1/10.000th of their peak values at the end of the waveguide, where the flux-regions have been placed. The width of the Gaussian wave in the frequency domain is $\triangle(\omega a / 2 \pi c)=0.1$.

The total bending loss is a mixture of losses at different stages. Losses incur at the entry and exit interfaces between the ridge waveguide and the corner element 


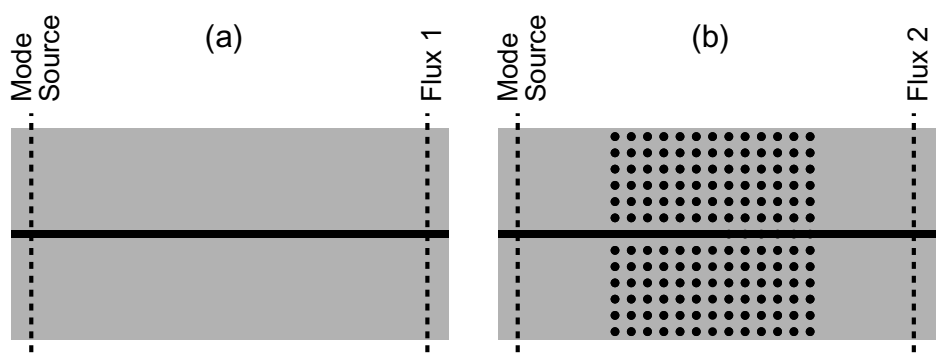

(c)

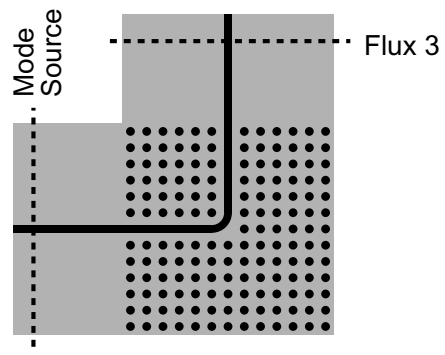

Fig. 4. The $z=0$ slice from (a) straight waveguide, (b) straight waveguide with the $2 \mathrm{D} \mathrm{PhC}$ but without the bend, and (c) SOI waveguide bend. The positions of the mode source and fluxes are as shown.

as well as at the $90^{\circ}$ bend in the corner element. In order to identify the losses for each stage, we compute the fluxes through various planes as shown in Fig. 4 . The overall transmission of the structure is found by comparing the transmission of the SOI waveguide bend and a straight ridge waveguide of the same length. It can be
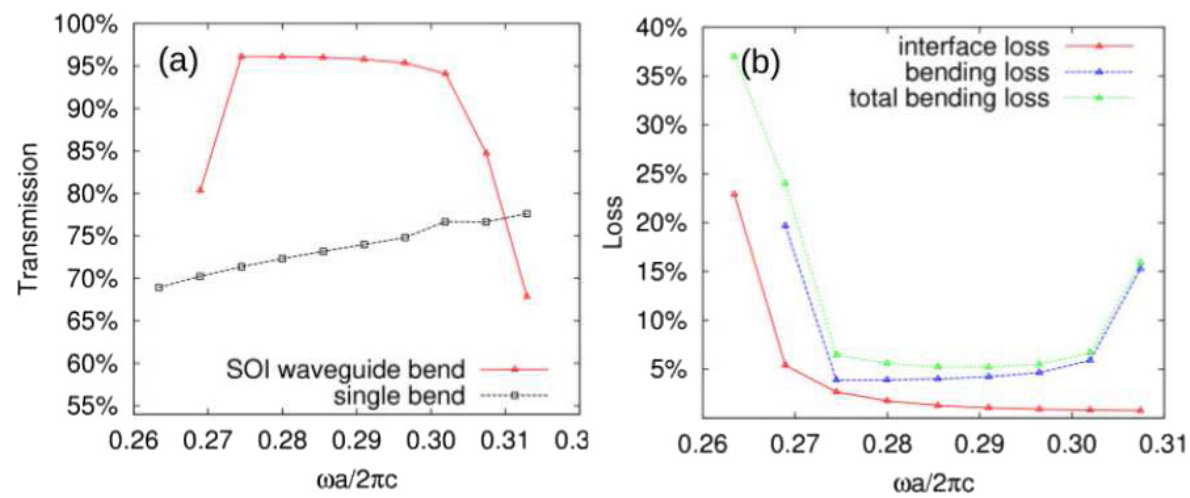

Fig. 5. (Color online) (a) Transmissions through the bend as a function of the normalized frequency in the range $0.26<\omega a / 2 \pi c<0.30$, for the SOI waveguide bend and single bend (here single bend is used to describe the ridge waveguide bend without photonic crystal assistance. (b) Various losses as a function of $\omega a / 2 \pi c$ for the photonic crystal assisted SOI waveguide bend. These results are for the case where the silicon elements are completely immersed inside silica with $\epsilon_{b}=2.25$. 
defined as, $T=P_{\text {flux } 3} / P_{\text {flux } 1}$, where flux1 and flux3 are measured at the locations shown in Figs. 4(a) and 4(c), respectively. The interface loss, i.e., the loss due only to reflections at the entry and exit interfaces can be defined as, $1-P_{\text {flux } 2} / P_{\text {flux } 1}$, where flux2 is shown in Fig. 4(b). The loss due only to the $90^{\circ}$ bend inside the corner element is $1-P_{\text {flux } 2} / P_{\text {flux } 3}$. Finally, the total bending loss of the entire structure is $1-P_{\text {flux } 3} / P_{\text {flux } 1}$.

The transmission and loss values are shown in Fig. 5. Figure 5(a) shows the transmission of the SOI waveguide bend as a function of frequency with red filled

(a)

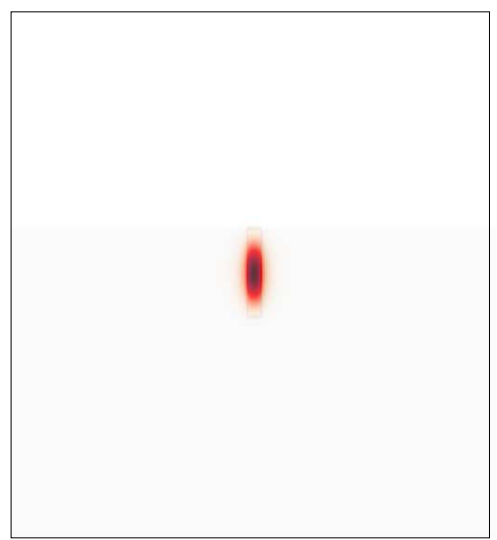

(c)

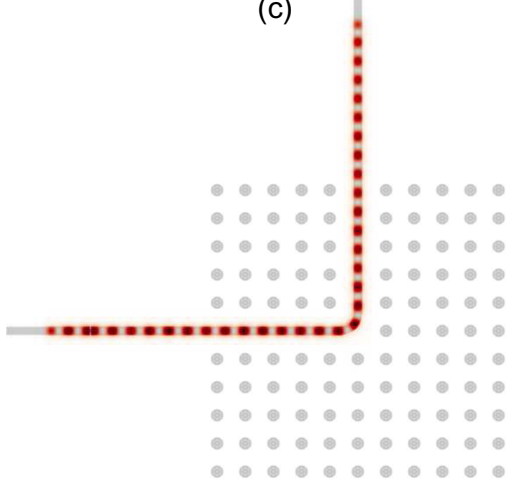

(b)

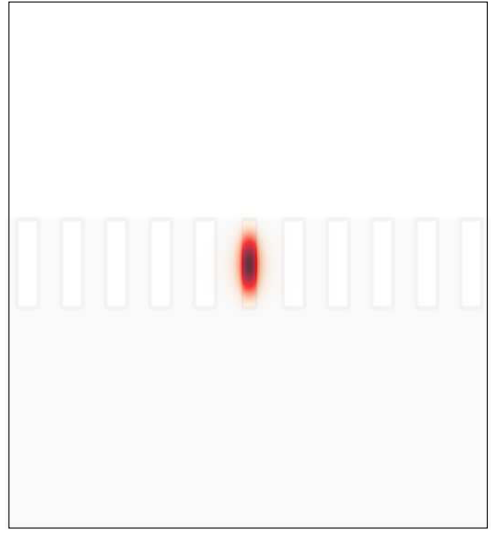

(d)

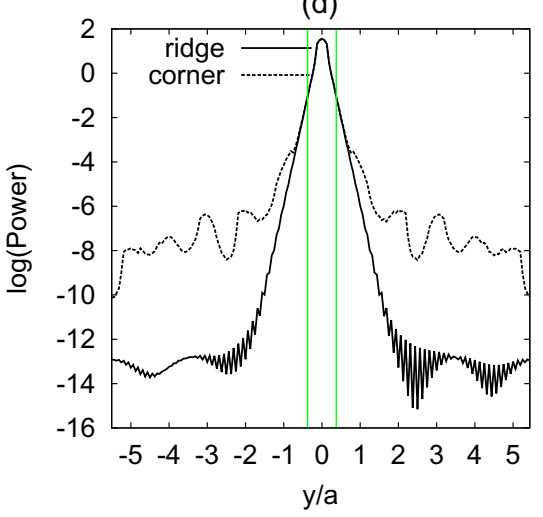

Fig. 6. (Color online) Various power snapshots calculated from the FDTD simulation at the operating frequency $\omega a / 2 \pi c=0.2897$. (a) Power profile of the ridge waveguide at $x=0$. (b) The power profile for the corner element inside the PhC. (c) Power snapshot at the $z=0$ plane. (d) The power profile along the $y$-axis at $z=0$ at fixed $x$. The solid curve is the power profile of the ridge waveguide (without $\mathrm{PhC}$ ). The dashed curve is the power profile of the ridge waveguide with the $2 \mathrm{D} \mathrm{PhC}$ cladding. The width of the ridge is indicated with green vertical lines. Note that the vertical axis is logarithmic. The two profiles are reasonably close, so there is very little reflection passing from the ridge waveguide into the corner element. The width of the ridge is indicated with green vertical lines. Note that the vertical axis is logarithmic. 
triangles. The transmission values for just the single bend without photonic crystal assistance are shown with black blank squares. The single bend consists of a single waveguide without $\mathrm{PhC}$ assistance.

Comparing the SOI waveguide bend with single bend, we can see that the contribution of $\mathrm{PhC}$ assistance is very significant, since this addon enhances the transmission by about $25 \%$, in the range $0.2745<\omega a / 2 \pi c<0.3075$. This range of frequencies partially overlaps the bandgap of the $2 \mathrm{D}$ square lattice $\mathrm{PhC}$ corner element. The overlap is not perfect as $2 \mathrm{D}$ square lattice, where the cylinders are infinitely long, is only an approximation to the present 3D structure where the height of the cylinders is finite. In Fig. 5(b), the obtained interface loss, bending loss and total bending loss, as a function of frequency are plotted with red filled triangles, blue filled triangles and with green filled triangles, respectively. While the major part of the total loss arises from bending, the loss at the two transitions at the interfaces is also measurable.

Next we present the FDTD simulation snapshots for some specific frequencies in Fig. 6. The chosen frequency $\omega a / 2 \pi c=0.2897$ is roughly in the middle of the range where transmission is maximum. Figure $6(\mathrm{a})$ is the profile snapshot in the $y z$ plane of the ridge waveguide. Figure $6(\mathrm{~b})$ shows the same profile inside the corner element, and Fig. 6(c) is the snapshot in the $z=0$ plane. Figure 6(d) compares the profiles of the modes in the ridge waveguide and in the corner element with $x$ fixed and $z=0$.

\section{Optimizing the Corner Element}

When a wave is incident on a photonic crystal of infinite size, if its frequency is within the bandgap of the photonic crystal, the wave cannot propagate inside the crystal. Nevertheless, because the propagation vector $\beta$ would be complex at a frequency inside the bandgap, it does penetrate a certain distance into the crystal, decaying exponentially inside. With a finite photonic crystal, the wave would "leak" at the other end if the thickness of the photonic crystal is not sufficiently long - a phenomenon akin to "barrier penetration." Since the main source of loss is due to the bend at the corner element, one needs a large $\mathrm{PhC}$ to minimize the leakage. On the other hand, one would obviously need a small corner element in any real-life application, say in an optical circuit where real estate would be at a premium. To determine the optimal size for the corner element, we varied the number of cylinders on each side of the ridge and repeated the transmission measurements for each case.

We consider the effect of increasing number of cylinders in the $2 \mathrm{D} \mathrm{PhC}$ on transmission. We varied the number of cylinders on either side of the ridge and computed the transmission for each case, as shown in Fig. 7. As would be expected, transmission increases with an increase in the number of cylinders, but with diminishing returns, reaching a more or less steady value after about seven cylinders on each side. We attribute the small loss to the less-than-perfect coupling of the modes before and after the $90^{\circ}$ turn inside the $\mathrm{PhC}$. 


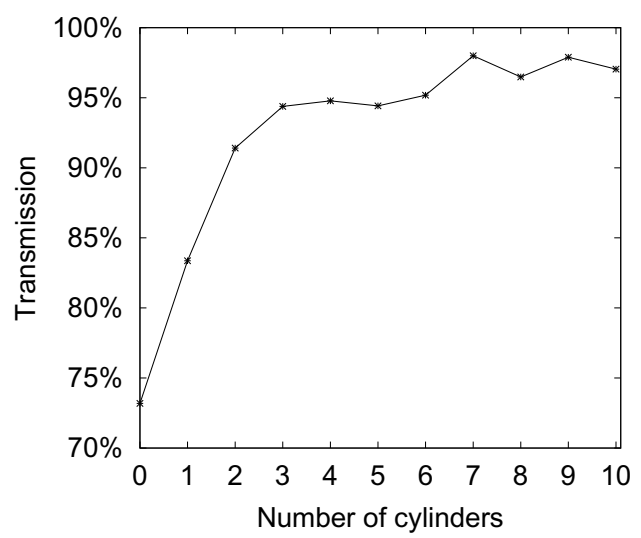

Fig. 7. Transmission versus number of cylinders in the corner element.

Throughout this study, we adopted a compromise and fixed the number of cylinders at 5 on each side of the ridge.

\section{Comparison of $2 \mathrm{D}$ and $3 \mathrm{D}$ Calculations}

3D FDTD calculations require considerably higher computational resources than $2 \mathrm{D}$ calculations, both in memory and CPU time. Therefore, it is important to understand the added benefit of the extra computational burden. Although, in general, the degree of discrepancy between the two approaches would certainly depend on the particular problem at hand, it would be illuminating to see how closely the $2 \mathrm{D}$ problem approximates the $3 \mathrm{D}$ problem in our case. In the $2 \mathrm{D}$ problem, the transmission through the $90^{\circ}$ bend peaks exactly at the bandgap of the $2 \mathrm{D}$ square

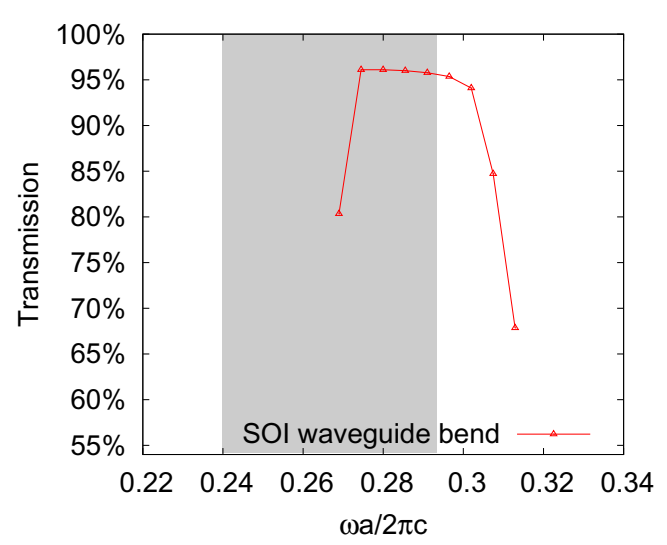

Fig. 8. (Color online) The transmission of the SOI waveguide bend using fully 3D FDTD method. The gray region marks the bandgap of the perfect $2 \mathrm{D}$ square lattice with the same $2 \mathrm{D}$ geometry. There is only a partial overlap of the high transmission frequency range with the photonic bandgap of the $2 \mathrm{D}$ structure. 
lattice structure ${ }^{36}$ and diminishes rapidly for frequencies outside the bandgap. In our $3 \mathrm{D}$ calculations, we find the frequency range where the transmission through the band is maximum and the bandgap of the perfect $2 \mathrm{D}$ photonic crystal overlaps only partially. Whereas the bandgap of the perfect $2 \mathrm{D}$ square crystal is the range $0.24<\omega a / 2 \pi c<0.293$, the frequency range, where transmission is maximum for the $3 \mathrm{D}$ problem, is the much narrower region $0.273<\omega a / 2 \pi c<0.302$, as displayed in Fig. 8. Thus, we conclude that, although the $2 \mathrm{D}$ calculations can be used as a rough starting point, fully $3 \mathrm{D}$ calculations are needed for the actual design.

\section{Conclusion}

We investigated a slab waveguide/bend system mounted on a silica substrate that would effectively guide light with low loss. We considered silica-silicon structure for the guiding slab, and found a transmittance value of over $95 \%$ for TE-like modes with a $11 \times 112 \mathrm{D} \mathrm{PhC}$ corner element. Increasing the size of the corner element led to slight improvements in transmittance. We performed fully $3 \mathrm{D}$ frequency domain band structure calculations and real time FDTD calculations to determine the losses of the structure.

Our objective was to demonstrate the efficacy of the photonic crystal corner element in drastically reducing the losses at the sharp corner. Although low losses can also be achieved without the photonic crystal assistance by using a larger bending radius, we believe our results are significant as they demonstrate the validity of photonic crystal assistance without having to resort to making any approximations such as using $2 \mathrm{D}$ calculations with "effective" values for the dielectric constants. We find that while the 2D structure can be used as a rough initial guide, the fully $3 \mathrm{D}$ treatment of the problem yields results quite different from those predicted solely by using $2 \mathrm{D}$ calculations.

\section{Acknowledgments}

The authors wish to acknowledge partial support from TÜBITAK, Turkish Scientific and Technological Research Agency. The numerical calculations reported in this paper were partly performed at TÜBITAK ULAKBIM, High Performance and Grid Computing Center.

\section{References}

1. R. A. Soref, J. Schmidtchen and K. Petermann, J. Quant. Electron. 27, 1971 (1991).

2. A. G. Rickman and G. T. Reed, IEE Proc. Optoelectron. 141, 391 (1994).

3. M. Tokushima et al., Appl. Phys. Lett. 76, 952 (2000).

4. T. Sndergaard, J. Arentoft and M. Kristensen, J. Lightwave Technol. 20, 1619 (2002).

5. T. Baba et al., IEEE J. Quant. Electron. 38, 743 (2002).

6. D. Sun et al., J. Lightwave Technol. 27, 4610 (2009).

7. N. Eti and H. Kurt, J. Quant. Electron. 52, 1 (2016).

8. A. Mekis et al., Phys. Rev. Lett. 77, 3787 (1996). 
9. M. Bayindir et al., Phys. Rev. B 63, 1 (2001).

10. H. Kurt, I. H. Giden and K. Ustun, J. Opt. Soc. Amer. B 28, 495 (2011).

11. H. S. Sozuer, J. W. Haus and R. Inguva, Phys. Rev. B 45, 13962 (1992).

12. H. S. Sozuer and J. P. Dowling, J. Mod. Opt. 41, 231 (1994).

13. S. Noda et al., Science 289, 604 (2000).

14. M. Imada et al., Appl. Phys. Lett. 88, 171107 (2006).

15. S. Kawashima, K. Ishizaki and S. Noda, Opt. Exp. 18, 386 (2010).

16. K. Ishizaki et al., Nat. Photon. 7, 133 (2013).

17. J. Cai et al., Appl. Opt. 43, 4244 (2004).

18. A. E. Erol and H. S. Sözüer, Opt. Exp. 23, 32690 (2015).

19. Y. Liu et al., Opt. Lett. 43, 2482 (2018).

20. A. Chutinan, M. Okano and S. Noda, Appl. Phys. Lett. 80, 1698 (2002).

21. A. Chutinan and S. Noda, Phys. Rev. B 62, 3388 (2000).

22. S. Johnson et al., Phys. Rev. B 62, 8212 (2000).

23. A. Chutinan, S. John and O. Toader, Phys. Rev. Lett. 90, 123901 (2003).

24. D. Zhang and Z. Feng, Opt. Int. J. Light Electron Opt. 124, 6298 (2013).

25. M. Loncar et al., J. Lightwave Technol. 18, 1402 (2000).

26. T. Stomeo et al., Opt. Quant. Electron. 37, 229 (2005).

27. S. Johnson et al., Phys. Rev. B 60, 5751 (1999).

28. S. Kuchinsky et al., Opt. Commun. 175, 147 (2000).

29. H. Taniyama, M. Notomi and Y. Yoshikuni, Phys. Rev. B 71, 153103 (2005).

30. H. S. Sözüer and K. Sevim, Phys. Rev. B 72, 195101 (2005).

31. S. Hughes et al., Phys. Rev. Lett. 94, 033903 (2005).

32. E. Kuramochi et al., Phys. Rev. B 72, 161318 (2005).

33. A. Taflove and S. C. Hagness, Computational Electrodynamics: The Finite-Difference Time-Domain Method (Artech, Norwood, MA, 2000).

34. A. F. Oskooi et al., Comput. Phys. Commun. 181, 687 (2010).

35. S. G. Johnson and J. D. Joannopoulos, Opt. Expr. 8, 173 (2001).

36. H. S. Sözüer and H. D. Şengün, Int. J. Mod. Phys. B 25, 2167 (2011). 